\title{
Spectral selective fluorescence molecular imaging with volume holographic imaging system
}

\author{
Yanlu Lv*, Jiulou Zhang*, Fei Liu*, Junwei Shi*, \\ Huizhi Guang*, Jing Bai* and Jianwen Luo, ${ }^{*, *}$ \\ *Department of Biomedical Engineering \\ School of Medicine, Tsinghua University \\ Beijing 100084, P. R. China \\ ${ }^{\dagger}$ Center for Biomedical Imaging Research \\ Tsinghua University \\ Beijing 100084, P. R. China \\ tuo_jianwen@tsinghua.edu.cn
}

Received 24 June 2015

Accepted 28 September 2015

Published 26 November 2015

\begin{abstract}
A compact volume holographic imaging (VHI) method that can detect fluorescence objects located in diffusive medium in spectral selective imaging manner is presented. The enlargement of lateral field of view of the VHI system is realized by using broadband illumination and demagnification optics. Each target spectrum of fluorescence emitting from a diffusive medium is probed by tuning the inclination angle of the transmission volume holographic grating (VHG). With the use of the single transmission VHG, fluorescence images with different spectrum are obtained sequentially and precise three-dimensional (3D) information of deep fluorescent objects located in a diffusive medium can be reconstructed from these images. The results of phantom experiments demonstrate that two fluorescent objects with a sub-millimeter distance can be resolved by spectral selective imaging.
\end{abstract}

Keywords: Volume holographic grating; diffusive medium; fluorescence molecular imaging.

\section{Introduction}

Fluorescence molecular imaging has been explored as an effective noninvasive technique in the study of tissue organization and biological understanding of disease for its high performance, low cost and targeting selectivity. ${ }^{1}$ Many three-dimensional (3D) fluorescence imaging modalities have been proposed over the past few years such as laser-scanning microscopy, confocal microscopy and Fresnel incoherent correlation holographic imaging. Although these fluorescence microscopy techniques achieved

\$Corresponding author.

This is an Open Access article published by World Scientific Publishing Company. It is distributed under the terms of the Creative Commons Attribution 4.0 (CC-BY) License. Further distribution of this work is permitted, provided the original work is properly cited. 
good resolution, the imaging depth is quite limited due to the highly scattering and absorption nature of photons propagating through a diffusive medium..$^{2,3}$ Fluorescence molecular tomography is an outstanding technique in detecting fluorescent target immersed in the diffusive medium. ${ }^{4}$ However, when the fluorophores are closely situated within scattering medium, the precise reconstruction of the fluorophores from acquired fluorescence images becomes challenging, because the scattering property of the medium leads to a highly ill-posed inverse problem that can reduce the spatial resolution of reconstruction. ${ }^{5}$

Multi-spectral fluorescence imaging is considered as an effective way to improve the $3 \mathrm{D}$ reconstruction of underlying fluorescence source. ${ }^{6,7} \mathrm{~A}$ variety of commercially available components have been developed to obtain multi-spectral fluorescence emitted from the object, such as filter wheel, acousto-optic tunable filter (AOTF), liquid crystal tunable filter (LCTF), and thin film tunable filter (TFTF). Filter wheel is the most common method to perform spectral selective imaging, but it has a complex mechanical and optical structure. The switching speed of LCTF is typically several tens of milliseconds, and that of the AOTF is in the range of microseconds. However, both filter technologies suffer from the polarization-dependent property, which leads to poor light transmission efficiency (approximately 40\%) and the additional drivers make the imaging system more complex and expensive. ${ }^{8,9}$ As a newly developed spectral selecting technique, TFTF has sharp edge cut-offs and high out-of-band optical densities. But TFTF needs an array of thin film tunable filters to cover a wide wavelength range, because each single TFTF has a limited bandwidth. ${ }^{10}$

Holographic components, for example the volume holographic gratings (VHGs), have been successfully applied to a variety of $3 \mathrm{D}$ imaging applications such as the long working distance surface profilometry, ${ }^{11}$ the volume grating based confocal microscopes, ${ }^{12}$ and the real-time $3 \mathrm{D}$ imaging with multiplexed volume holographic grating. ${ }^{13}$ VHG is manufactured by recording the $3 \mathrm{D}$ interference patterns of two or more mutually coherent beams into materials such as phenanthrenequinone doped poly-(methyl methacrylate) (PQ-PMMA) photopolymer, photo-thermal-refractive (PTR) glass, and dichromated gelatin (DCG). ${ }^{14-16}$ Due to the unique high angular/spectral selectivity, VHGs are capable of diffracting the Bragg-matched components of the incident beam with the efficiency close to $100 \%$. Many efforts have been made in applying VHG into imaging systems to determine the depth and intensity information of fluorescent objects in the volumetric environment. ${ }^{17} \mathrm{An} \mathrm{N}$ ocular volume holographic imaging (VHI) system combining several VHG sensors with computational algorithms has been proposed to improve the depth resolution of VHI system for imaging broadband fluorescence beads located in a transparent medium. ${ }^{18}$ The most recent example is the multi-depth VHI system. ${ }^{19-22}$ By exploiting the property of recording multiplex gratings in one volume, the multidepth VHI system can obtain both spatial and spectral information of the object in real time and with an imaging depth of $0.2 \mathrm{~mm}$.

In this paper, we present a compact and cost effective spectral selective VHI system. It consists of a demagnification system and a classic $4 \mathrm{~F}$ transmission geometry VHI system. With the help of single transmission VHG, the imaging system system can acquire fluorescence spectral information of the fluorescent objects located several millimeters in diffusive medium in a spectral selective imaging manner and resolve the sub-millimeter distance between the immersed fluorophores.

This paper is organized as follows. The basic principles of the spectral selective VHI system for detecting a variety of single-colored fluorescent objects in diffusive medium and the reconstruction algorithm are described in Sec. 2. The experimental setup and results are presented in Sec. 3. The performance of the spectral selective VHI system is discussed in Sec. 4. Finally, a summary of our work is presented in Sec. 5 .

\section{Principles}

The spectral selective VHI system is composed of two main parts: a demagnification imaging system and a $4 \mathrm{~F}$ transmission geometry VHI system. The $4 \mathrm{~F}$ transmission geometry VHI system was built with a single transmission VHG, which was recorded by two planar beams. Due to the Bragg selectivity of VHG, the lateral FOV of the $4 \mathrm{~F}$ transmission VHI system is a function of the bandwidth of broadband illumination and the numerical aperture (NA) of the VHI system. Because of the narrow bandwidth of illumination beam and 
the finite NA of objective lens, typical VHI system with fixed physical aperture can only obtain a narrow FOV. In our work, the lateral FOV of the spectral selective VHI system is enlarged by using broadband fluorescence illumination and the demagnification imaging system.

\subsection{Principles of $V H G$}

The imaging capability of VHG is based on the Bragg selectivity and degeneracy properties of volume holograms. For a transmission grating, the diffraction efficiency is proportional to the incident intensity ${ }^{23}$

$$
\eta \propto \sin c^{2}\left(\frac{L\left|\Delta \mathbf{k}_{z}\right|}{2 \pi}\right)
$$

where $\Delta \mathbf{k}_{z}$ is the Bragg mismatch factor, which is caused by angular or wavelength detuning from the Bragg matching condition.

Due to this Bragg selectivity, the diffraction peak efficiency can reach $100 \%$ under Bragg-matched condition in theory. The transmission VHG used here is recorded in a $2.1 \mathrm{~mm}$-thick crystal of PQPMMA material with a green color laser $\left(\lambda_{r}=514 \mathrm{~nm}\right)$ and has a $\sim 1$ inch clear aperture. It is provided by the Center for Optoelectronic Biomedicine, National Taiwan University. Experimentally measured diffraction intensity and the rigorous coupled wave simulation results of angular selectivity at $514 \mathrm{~nm}$ are shown in Fig. 1(a). The measured results are normalized by the Braggmatched diffraction intensity $I_{0}=I_{d}\left(34.3^{\circ}\right)$. Only the simulation result of spectral selectivity is provided in Fig. 1(b) for lacking of the wavelength tunable laser with narrow bandwidth (bandwidth $<1.0 \mathrm{~nm}$ ). In both the simulation and experiments, the thickness of the VHG is $2.1 \mathrm{~mm}$, the wavelength of the probe beam is $514 \mathrm{~nm}$, and the incident angle is $\theta_{p}=34.3^{\circ}$.

The wavelength degeneracy property offers VHG the capability of imaging within a wide wavelength range. When the VHG is probed at an appropriate angle, wavelength different from the recording beam can also be diffracted at its maximum efficiency. To illustrate the operation principle, a schematic that shows the procedure and the K-sphere diagram of probing the transmission VHG with different wavelengths are given in Figs. 2(a) and 2(b) respectively. Beams with different wavelengths are diffracted with high efficiency by rotating the VHG to the Bragg-matched angles. And the Bragg-matched angle can be calculated from Eq. (2).

$$
\theta_{p 1}=\phi-\cos ^{-1}\left[\frac{\lambda_{p 1}}{\lambda_{p_{2}}} \cos \left(\phi-\theta_{p 2}\right)\right],
$$

where $\theta_{p 1}$ and $\lambda_{p 1}$ represent the incident angle and wavelength of probing beam, $\theta_{p 2}$ and $\lambda_{p 2}$ represent the incident angle and wavelength of signal beam in recording geometry, and $\phi$ is the inclination of $\mathrm{K}$ vector of the VHG with respect to its corresponding hologram normal.

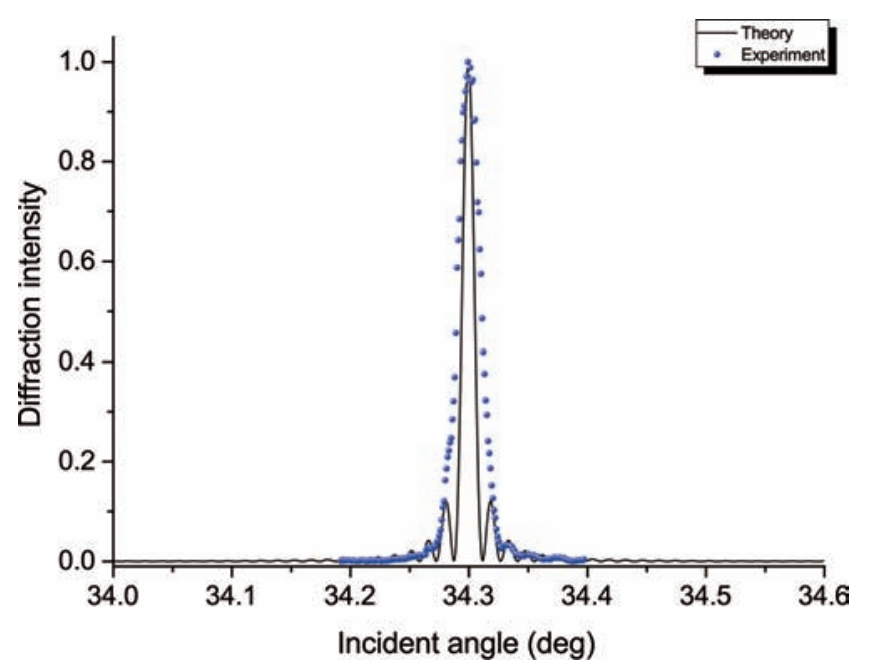

(a)

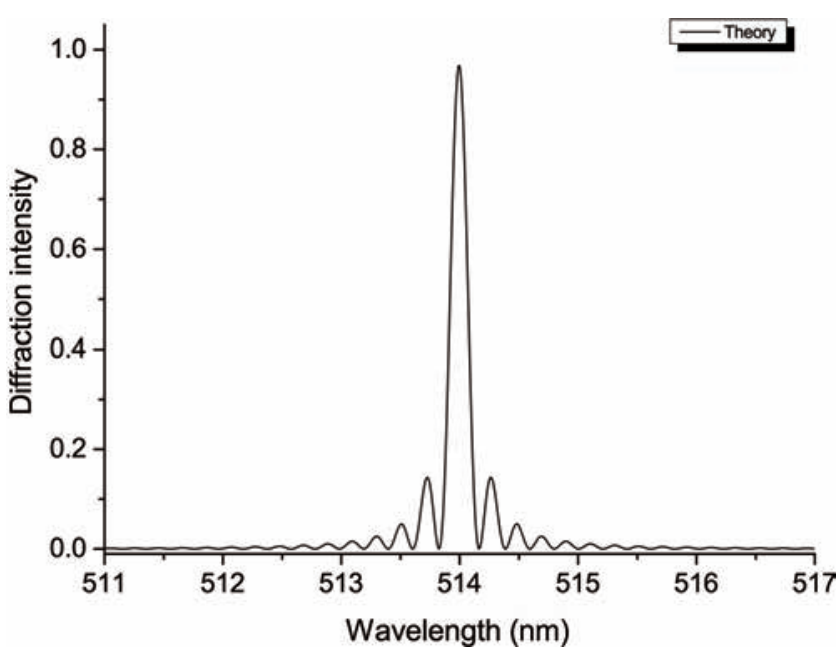

(b)

Fig. 1. (a) The normalized experimentally measured diffraction intensity and simulation result of the angular selectivity curve of the single transmission VHG. (b) Numerical simulation result of the wavelength selectivity of the single transmission VHG. 


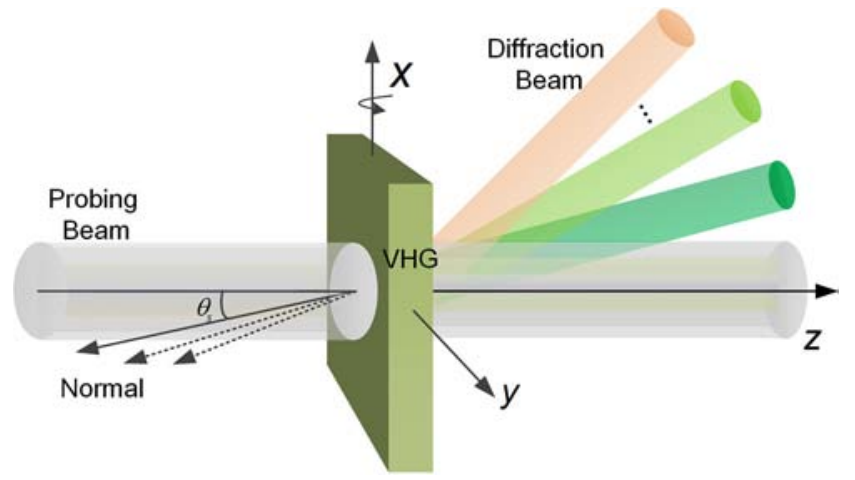

(a)

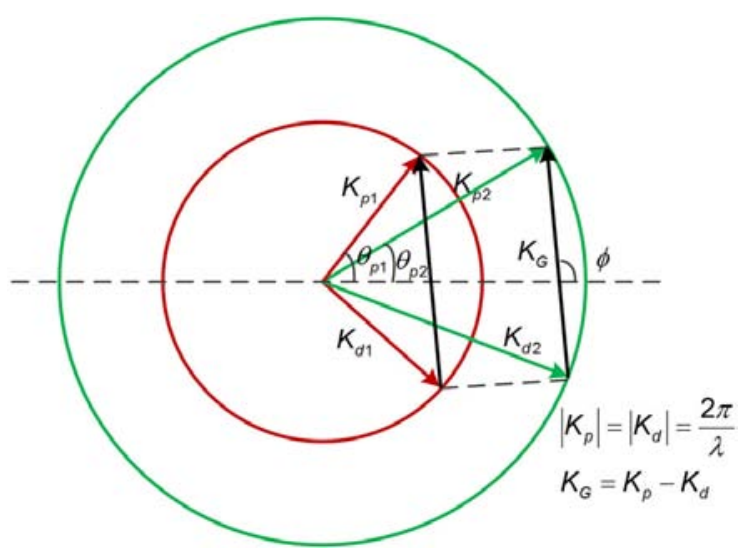

(b)

Fig. 2. (a) Schematic for illuminating VHG with collimated beam consisting of multiple wavelengths. The wavelength changes of the diffraction beam correspond to the angles between the normal vector of the VHG and the $z$-axis. The dash normal vectors indicate that the VHG is in different rotation positions around $x$-axis. (b) The K-sphere diagram for grating recording geometry with one wavelength (green) and probed by another wavelength (red). Note that small rotations around $x$-axis should be applied to the VHG to match Bragg condition for different wavelengths. $\theta_{p 1}$ and $\lambda_{p 1}$ represent the incident angle and wavelength of probing beam, respectively, $\theta_{p 2}$ and $\lambda_{p 2}$ represent the incident angle and wavelength of signal beam in recording geometry, respectively. $\phi$ is the inclination of $\mathrm{K}$-vector of holographic grating with respect to its corresponding hologram normal. $K_{p}, K_{d}$, and $K_{G}$ represent the $\mathrm{K}$-vector of probe beam, diffraction beam, and holographic grating, respectively.

\section{2. $\quad F O V$ of $\mathrm{VHI}$ system with demagnification optics under fluorescence illumination}

The imaging system utilizes the Bragg selectivity and degeneracy properties of VHG to acquire broadband fluorescence images. The schematic of illuminating the $4 \mathrm{~F} \mathrm{VHI} \mathrm{system} \mathrm{with} \mathrm{a} \mathrm{point} \mathrm{source}$ is shown in Fig. 3. The probing beam is used as the illumination of the VHI system. A transmission VHG, recorded with wavelength $\lambda_{r}$, is placed on the Fourier plane between the objective lens and the collector lens. The collector lens is placed behind the VHG to map the diffracted beam onto a charge-

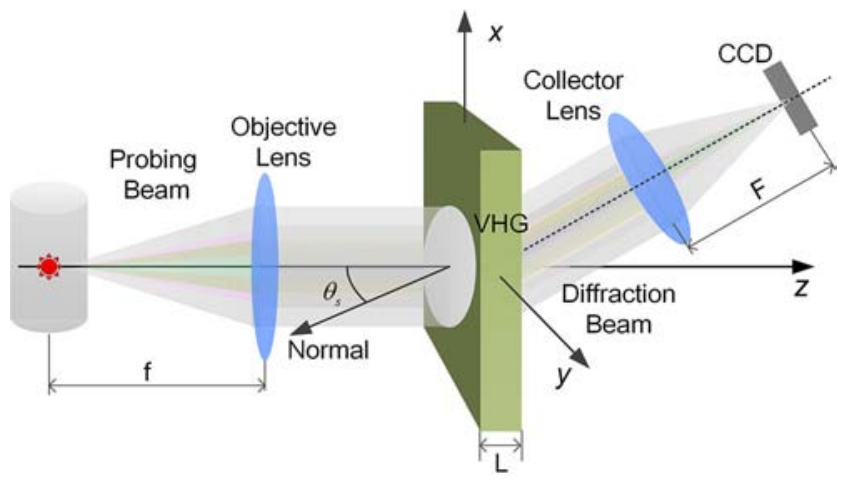

Fig. 3. Schematic of the $4 \mathrm{~F}$ transmission geometry VHI system. $L$ is the thickness of the VHG, $f$ and $F$ are the focal length of the objective lens and the collector lens. coupled device (CCD). When the system is probed with the monochromatic illumination, a corresponding slit pattern is mapped onto the detector, so the resulting diffraction pattern of broadband illumination is a superposition of slit patterns corresponding to different monochromatic spectrum.

Since the Bragg wave-vector pair only allows the volume hologram to diffract the Bragg-matched component of the probing beam and image a slit pattern on the image plane. When the wavelength of probing beam is different from the recording one, the Bragg angle-wavelength coupling degeneracy would require a displacement of the point source in the $x$ direction by an amount of $\Delta x$ to meet the Bragg condition. The relationship between the lateral FOV,$\Delta \lambda$ and $\lambda_{r}$ is given in Eq. (3) under paraxial condition.

$$
F O V_{x}=\frac{2 D \theta_{s} \Delta \lambda}{N A_{V H I S} \lambda_{r}}
$$

where $F O V_{x}$ is the lateral FOV, $N A_{V H I S}$ is the NA of the VHI system, and $D$ is the diameter of the objective lens.

The schematic shown in Fig. 4 is the imaging system, which is used to detect single fluorescence spectrum objects located in a diffusive medium in spectral selective imaging manner. A pair of achromatic lenses (AC508-100-A, AC254-030-A, 


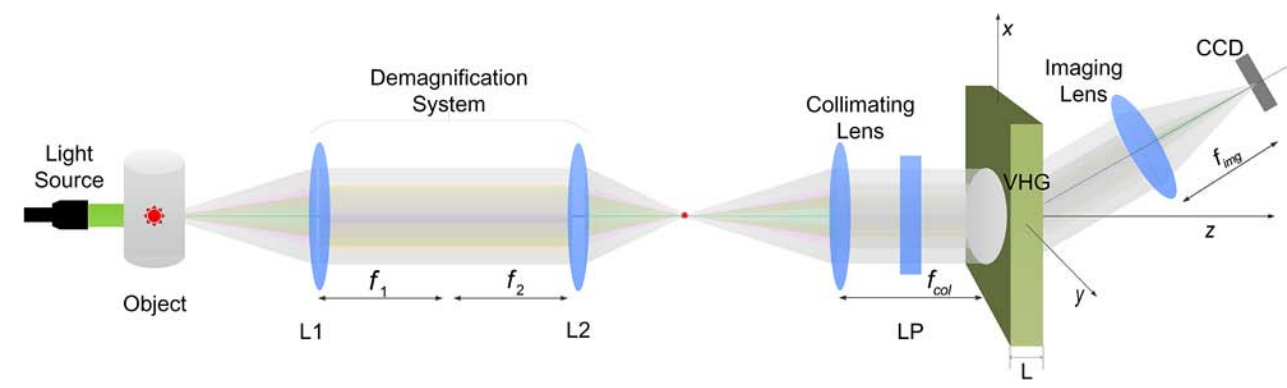

Fig. 4. Schematic of the spectral selective VHI system for detecting fluorescent objects within diffusive medium. Light source provides uniform excitation illumination in the transmission mode. The fluorescence emitting from the fluorescent object in the phantom is collected through the demagnification system and forms a real image on the detector, CCD, after being diffracted by VHG. LP: long-pass filter.

Thorlabs, NJ, US) with $f_{1}=100 \mathrm{~mm}$ and $f_{2}=$ $30 \mathrm{~mm}$ is used to build the demagnification imaging system. Another pair of achromatic lenses (AC254100-A, AC254-075-A, Thorlabs, NJ, US) with $f_{\text {col }}=100 \mathrm{~mm}$ and $f_{\text {img }}=75 \mathrm{~mm}$, and a CCD camera (Clara, Andor Technologies, Belfast, Northern Ireland) form the $4 \mathrm{~F}$ transmission geometry VHI system. The optical field emitted by the fluorophores located in diffusive medium is transformed by the combination of lenses and subsequently diffracted by the VHG. In the configuration of the experiments, the imaging object appears on the front focal plane of lens L1. When the object is illuminated by a plane wave, the fluorescence emitted from the fluorophores within diffusive medium is collected by the demagnification lens pair (L1 and L2). And a spatially demagnified image is acquired on the front focal plane of collimating lens.

To fit the whole diameter of the phantom into the lateral FOV, the 10:3 demagnification lens pair is placed in front of collimating lens of the classic $4 \mathrm{~F}$ transmission VHI system. To verify the improvement of lateral FOV, both the classic $4 \mathrm{~F}$ transmission VHI system and the spectral selective VHI system with demagnification system are experimentally measured [Fig. 5]. The test target was illuminated with the broadband light source (FWHM spectral bandwidth $\sim 34 \mathrm{~nm}$ ) which was acquired with a fluorescence filter (FF01-542/27-25, Semrock, NY, US) and Xe lamp (Max 302, Asahi Spectra, Tokyo, Japan). When the VHG is illuminated with a broadband probing beam, the lateral FOV is larger than $4.0 \mathrm{~mm}$, and the calculated value with Eq. (3) is $3.93 \mathrm{~mm}$.

With the demagnification optics, the spectral selective VHI system achieves a three times larger lateral FOV than only uses the classic $4 \mathrm{~F}$ transmission geometry VHI system. However, the trade-off is the degradation of the spatial resolution.

\subsection{Tomographic reconstruction with Tikhonov regularization}

In continuous wave domain, the diffusion equation coupled with Robin-type condition is a widely used mathematical model to describe the photon propagation in a diffusive medium. ${ }^{24}$

$$
\begin{cases}-\nabla[D(r) \nabla G(r)]+\mu_{a}(r) G(r)=S(r) & r \in \Omega \\ G(r)+2 D(r) q \frac{\partial G(r)}{\partial \mathbf{n}}=0 & r \in \partial \Omega\end{cases}
$$

where $D(r)$ denotes the diffusion coefficient at position $r$, and $\mu_{a}$ is the absorption coefficient. $G(r)$

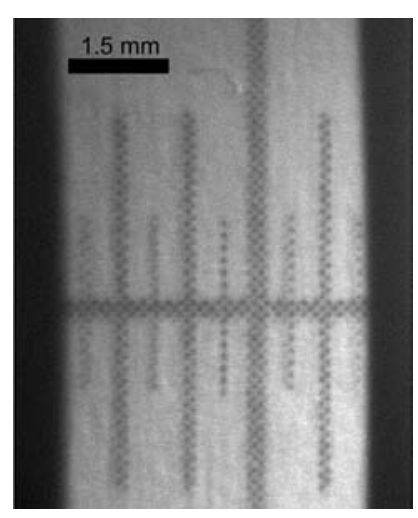

(a)

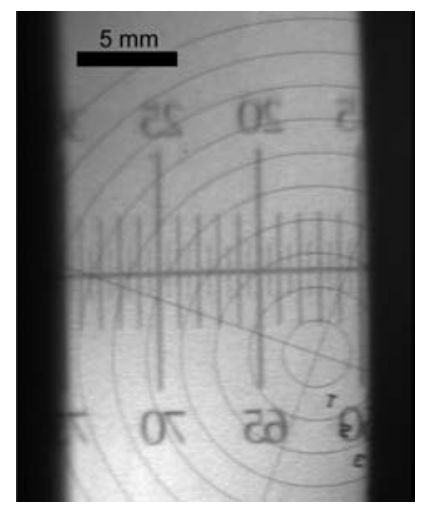

(b)
Fig. 5. Lateral FOV of (a) the classic $4 \mathrm{~F}$ transmission VHI system and (b) the VHI system with demagnification system. The test target is illuminated with a broadband light source (FWHM spectral bandwidth $\sim 34 \mathrm{~nm}$ ). The lateral FOV is enlarged more than three times with the demagnification system. 
and $S(r)$ are the Green's function and continuous wave source term, respectively. $\nabla$ denotes grad operator and $\mathbf{n}$ is the outward normal vector of the surface. $\Omega$ is the domain of the imaging object with a boundary of $\partial \Omega$, and $q$ is coefficient related to the internal reflection at the boundary.

In this paper, the diffusion equation is solved using Kirchhoff approximation (KA) method. ${ }^{25}$ And then, the inverse problem is described in the form of linear system matrix equation

$$
\Phi=W X,
$$

where $X$ is the distribution of the unknown fluorescent object to be reconstructed, $W$ is a weight matrix describing the maps between the unknown distribution of fluorescent object and the measured fluorescent data, and $\Phi$ is a column vector containing the fluorescent measurements of all perspectives. In order to overcome the ill-posedness of linear system matrix equation, L2-norm regularization term is introduced in the optimization function:

$$
\underset{X \geq 0}{\operatorname{argmin}}\|W X-\Phi\|_{2}+\alpha\|X\|_{2},
$$

where $\alpha$ is the regularization parameter. Tikhonov regularization method is used to obtain its minimalnorm least-squares solution iteratively. ${ }^{26}$ With the generalized inverse of matrices, the minimization of Eq. (6) can be solved as follows

$$
X_{k}=X_{k-1}-\left(W^{-1} W+\alpha I\right)^{-1} W^{-1}\left(W X_{k-1}-\Phi\right),
$$

where $k$ is the number of iterations, and $I$ is an identity matrix.

\section{Experimental Results}

\subsection{Experimental setup and data acquisition}

To evaluate the performance of the spectral selective VHI system in detecting different kinds of single-colored fluorescent objects in a diffusive medium, two cylindrical transparent glass phantoms (diameter $10.0 \mathrm{~mm}$, height $50.0 \mathrm{~mm}$ ) filled with 1\% Intralipid were prepared. Quantum dots (QDs) with different emission peak wavelengths can be excited with the same excitation wavelength and exhibit steep spectral slopes, so QDs can be detected more easily with spectroscopic method compared to other fluorophores. Two kinds of QDs (PL-QDN-530 and PL-QDN-620, PlasmaChem,
Germany), which emit at a peak wavelength of $530 \pm 20 \mathrm{~nm}$ (green) and $620 \pm 20 \mathrm{~nm}$ (red), respectively, were chosen as the fluorescence probe. Both the green and red hydrosoluble QDs powers were dissolved into $1.0 \mathrm{mg} / \mathrm{mL}$ aqueous solution respectively. The inclinations of the transmission VHG were $36.066^{\circ}$ and $42.694^{\circ}$, for acquiring fluorescence images of the green and red QDs.

Capillary quartz tubes (diameter $1.0 \mathrm{~mm}$ ) were used to fabricate the fluorophores within the phantom [Fig. 6]. In the first phantom, the first capillary tube filled with $\sim 1 \mu L$ red QDs solution was planted in the center of the phantom, and the depth to the boundary of the phantom was $5.0 \mathrm{~mm}$. The second tube filled with $\sim 1 \mu L$ green QDs solution was placed beside the first one and the edgeto-edge distance of the two fluorescence objects was $0.8 \mathrm{~mm}$. In the second phantom, the capillary tube filled with $\sim 1 \mu L$ green QDs solution and the one filled with $\sim 1 \mu L$ red QDs solution were placed $2 \mathrm{~mm}$ away from the boundary. And the edgeto-edge distance of the two capillary tubes was $0.8 \mathrm{~mm}$. The two phantoms were illuminated with a $488 \mathrm{~nm}$ laser in sequence for acquiring fluorescence images.

Before acquiring fluorescence images, the spectral selective VHI system was calibrated with a tunable laser (Argon Ion 543 AP-A01, Melles Griot, CA, US) and each rotational angle of VHG were calculated from Eq. (2) in advance to guarantee that each target wavelength can meet the Bragg condition. The inclination angles of the grating for different target fluorescence spectrum were sequentially switched during data acquisition.

During the acquisition of fluorescence images, uniform illumination was provided by the tunable laser. The fluorescence originating from the objects with the peak wavelengths around $530 \mathrm{~nm}$ and $620 \mathrm{~nm}$ was filtered by a $500 \mathrm{~nm}$ long-pass filter (FEL0500, Thorlabs, NJ, US) and collected by spectral selective VHI system. For $360^{\circ}$ full perspective imaging, the imaging object was rotated about its axis with an angular separation of $10^{\circ}$ with the aid of a computer-controlled rotation stage. The rotation of the transmission VHG was excuted through a compact rotation stage (URS50BPP, Newport, CA, US). The CCD camera was set to $4 \times 4$ pixel binning for detecting the diffracted fluorescence. 36 white-light images were captured with an angular separation of $10^{\circ}$ for $3 \mathrm{D}$ profile configuration of the phantom. ${ }^{27}$ 


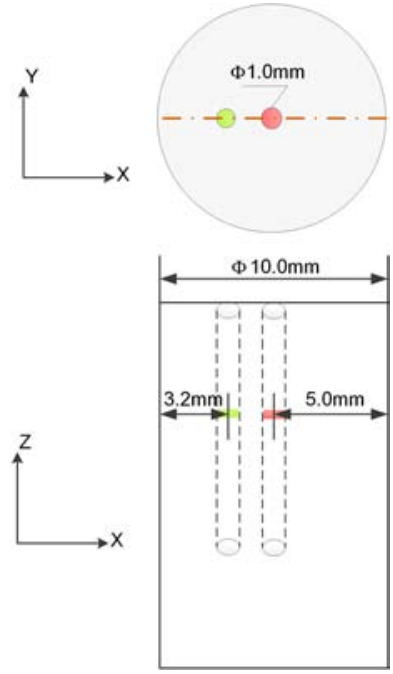

(a)
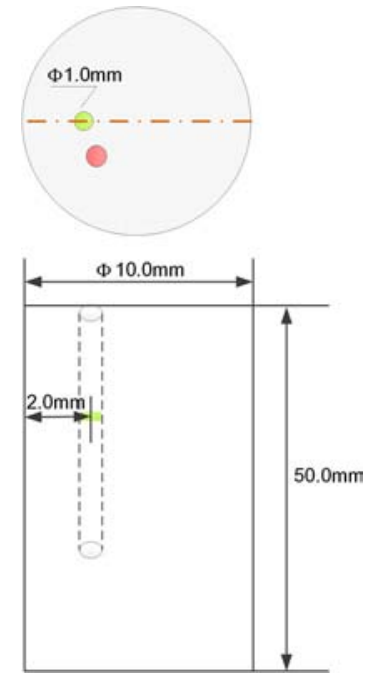

(b)

Fig. 6. Illustration of the phantom experimental setup. (a) The cross-section of the fluorophores in the first phantom and vertical section along the brown dashed dotted line. (b) The cross-section of the fluorophores in the second phantom and vertical section along the brown dashed dotted line. The two phantoms are cylindrical glass tubes (out diameter $10.0 \mathrm{~mm}$ ) containing $1 \%$ Intralipid. Capillary quartz tube (outer diameter $\sim 1.0 \mathrm{~mm}$ ) filled with $\sim 1 \mu L$ red QDs solution (the pink circular spot) and the capillary quartz tube filled with $\sim 1 \mu L$ green QDs solution (the green circular spot) were positioned at different depth within the diffusive medium.

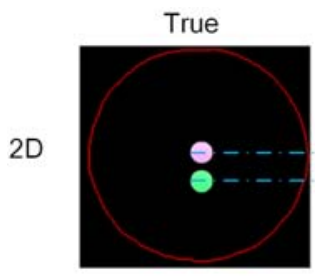

(a)

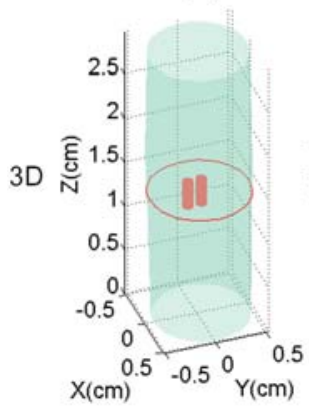

(e)

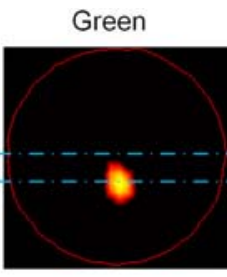

(b)

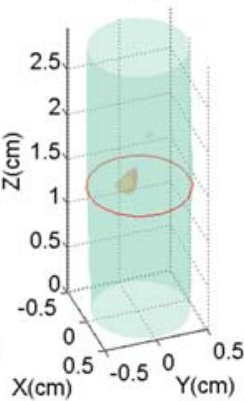

(f)

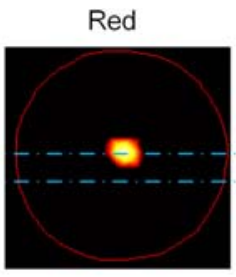

(c)

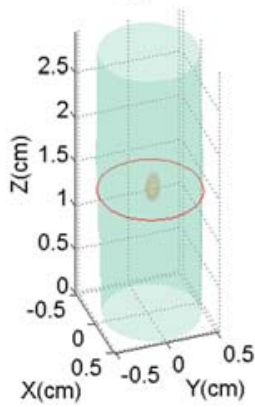

(g)

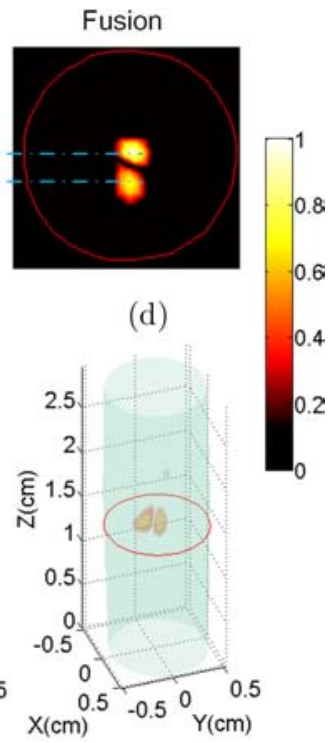

(h)

Fig. 7. Cross-sections and 3D reconstruction results of single-colored fluorescent objects. (a)-(d) Cross-sections at the height of $\mathrm{Z}=1.3 \mathrm{~cm}$. (a) Real position of the two single-colored fluorescent objects in the phantom. A capillary quartz tube that contains red quantum dot solution is placed at the center of the phantom (pink spot), and another tube that contains green quantum dots is placed $0.78 \mathrm{~mm}$ beside the center one (green spot). Reconstruction results with measurements at the center wavelength of (b) $530 \mathrm{~nm}$ and (c) $620 \mathrm{~nm}$, respectively. (d) Fusion image of the reconstructed objects at $530 \mathrm{~nm}$ and $620 \mathrm{~nm}$. (e) Real positions of the two single-colored fluorescent objects in three dimensions. Reconstructed 3D objects with measurements at the center wavelength of (f) $530 \mathrm{~nm}$ and (g) $620 \mathrm{~nm}$, respectively, and (h) the fusion result of (f) and (g). The geometric centroids of both the reconstructed objects and the real objects are depicted with two blue dashed lines. The separation between the two reconstructed capillary tubes is calculated to be $0.59 \mathrm{~mm}$. The red circles on the cross-sections indicate the phantom boundary, while the red circles on the $3 \mathrm{D}$ images indicate the position of cross-sections. 


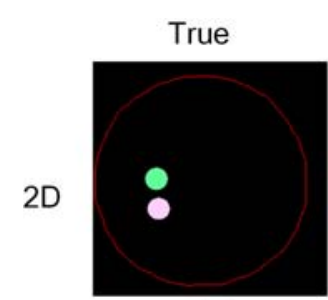

(a)

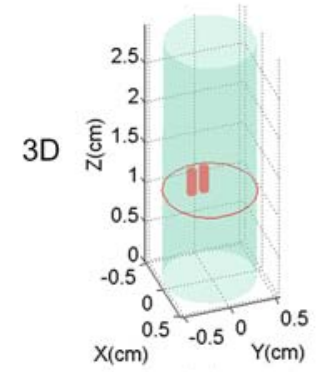

(e)

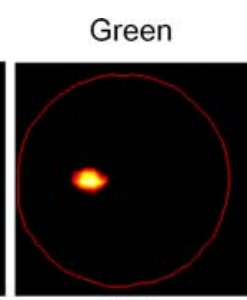

(b)

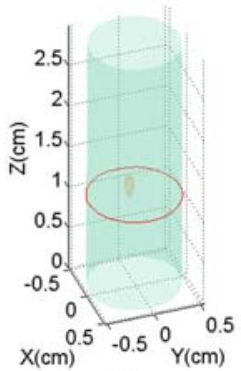

(f)

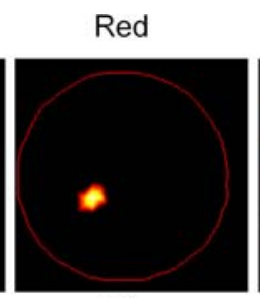

(c)

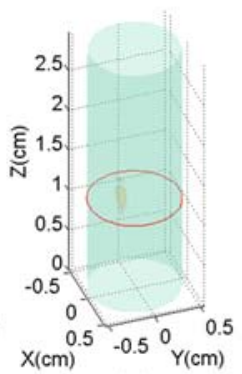

(g)

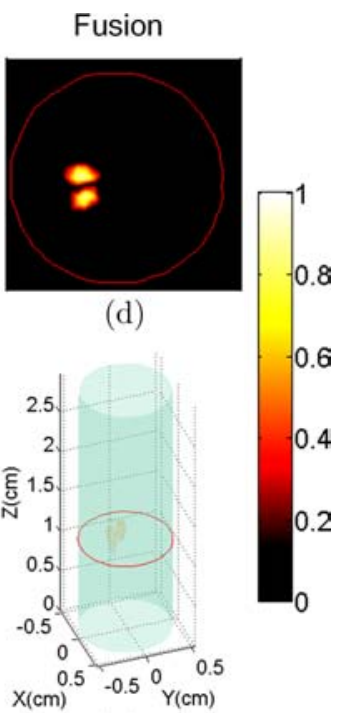

(h)

Fig. 8. Resconstruction results of the two single-colored fluorescent objects planted $2.0 \mathrm{~mm}$ to the boundary. (a)-(d) Cross-sections at the height of $Z=1.1 \mathrm{~cm}$. (a) Real positions of the two single-colored fluorescent objects in the phantom. The distance between the two capillary quartz tubes is $0.8 \mathrm{~mm}$. Reconstruction results with measurements at the center wavelength of (b) $530 \mathrm{~nm}$ and (c) $620 \mathrm{~nm}$, respectively. (d) Fusion image of the reconstructed objects at 530 and $620 \mathrm{~nm}$. (e) Real positions of the two single-colored fluorescent objects in three dimensions. Reconstructed 3D objects with measurements at the center wavelength of (f) $530 \mathrm{~nm}$ and (g) $620 \mathrm{~nm}$, respectively, and (h) the fusion result of $(\mathrm{f})$ and $(\mathrm{g})$. The red circles on the cross sections indicate the phantom boundary, while the red circles on the $3 \mathrm{D}$ images indicate the position of cross sections.

\subsection{Results}

The resconstruction results of the first phantom experiment are shown in Fig. 7. Figure 7(a) shows the true position of the two capillary tubes in the diffusive medium. Figures $7(\mathrm{~b})$ and $7(\mathrm{c})$ show the 2D reconstruction results of $530 \mathrm{~nm}$ and $620 \mathrm{~nm}$, respectively. Figure $7(\mathrm{~d})$ is the fusion results of Figs. 7(b) and 7(c). In Fig. 7(d), the separation between the two reconstructed single-colored fluorescent objects is well resolved. The corresponding $3 \mathrm{D}$ reconstruction results of fluorescence objects are shown in Figs. $7(\mathrm{e})-7(\mathrm{~h})$. The real separation between the centers of the two capillary quartz tubes is $1.78 \mathrm{~mm}$. The geometric centroid of the reconstruction results of the two capillary quartz tubes is calculated to be $1.59 \mathrm{~mm}$. The reconstruction results show good agreement between the reconstructed objects and the real objects. According to the reconstruction results, the two closely planted fluorophores located in the diffusive medium were clearly resolved by using the proposed spectral selective VHI imaging system.

Figure 8 shows the reconstruction results of the fluorophores planted close to the boundary of the phantom. Both single-colored fluorescent objects can be reconstructed with a high spatial resolution. However, the reconstructed objects deviate slightly from the real positions, and this may be improved by using better algorithms such as the L1 regularization. ${ }^{31}$

\section{Discussion}

The results of phantom experiments indicate that with broadband fluorescence illumination, the proposed VHI system can achieve a large lateral FOV and perform spectral selective imaging of macroscopic objects. Combined with corresponding reconstruction algorithm, the imaging method can selectively detect the closely planted macroscopic fluorescent objects located in diffusive medium with sub-millimeter resolution.

The procedures of acquiring emitted fluoresence from the diffusive medium with the conventional fluorescence molecular imaging system and the spectral selective VHI system are quite different. In the conventional imaging system, different kinds of fluorescence are collected with imaging lens after being filtered by appropriate band-pass filters and directly forming the fluorescence image on the detector plane. While in the spectral selective VHI system, only the wavelengths of the fluorescence 
Spectral selective fluorescence molecular imaging with VHI system

beam that satisfied Bragg-matched condition can be diffracted. And the diffraction beam forms a corresponding fluorescence image onto the CCD through the collector lens.

Although the VHG exhibits perfect angular/ spectral selectivity, the diffraction curve is approximately flat due to the Bragg degeneracy, and the diffraction intensity does not fluctuate significantly around its mean value (about $11.3 \%$ ), with the fluorophores located $2 \mathrm{~mm}$ away the central position, under the broadband fluorescence illumination condition. The wavelength degeneracy of volume holograms extends the lateral FOV of VHI system, but the separation between the target wavelengths should be wide enough to reduce the crosstalk of the two spectrum which is brought by the wavelength degeneracy. In the proposed system, the separation between two target wavelengths should be larger than $40 \mathrm{~nm}$.

The rotation of VHG is executed through a compact precise rotation stage. The rotation stage provides precise and continuous $360^{\circ}$ motion and allows for rotating speeds up to $40.0^{\circ} / \mathrm{s}$ with a resolution of $0.00022^{\circ}$. Therefore, the wavelength switching speed of this imaging system is comparable to LCTF, while its spectral resolution is better than LCTF and AOTF. ${ }^{28}$ The imaging time cost of the system mainly depends on the integration time of the detector and the rotation time for full perspective imaging. The imaging rate of the CCD camera is set to be 0.5 frame/s to acquire fluorescence image with high contrast. The rotation stage for mounting the imaging object can provide rotating speeds up to $55.5 \mathrm{~ms} / \mathrm{deg}$. And the total time of obtaining 36 fluorescence projections for one spectrum is $92 \mathrm{~s}$. The imaging time cost can be grately reduced by adopting the off-the-shelf VHGs with high diffraction efficiency and reconstruction algorithms that require fewer projections. ${ }^{29}$

When wavelength switches, wavelength error might be introduced by the absolute accuracy of VHG mechanical rotation part. The relationship between the wavelength error and the absolute accuracy of the mechanical rotation part is given by ${ }^{30}$

$$
\frac{\lambda_{0}-\lambda_{1}}{\lambda_{0}}=1-\frac{\sin \left(\theta_{B}-\Delta \theta\right)}{\sin \left(\theta_{B}\right)},
$$

where $\lambda_{0}$ is the target wavelength, $\lambda_{1}$ is the actually achieved wavelength of the mechanical rotation, $\theta_{B}$ is the Bragg-matched incident angle corresponding

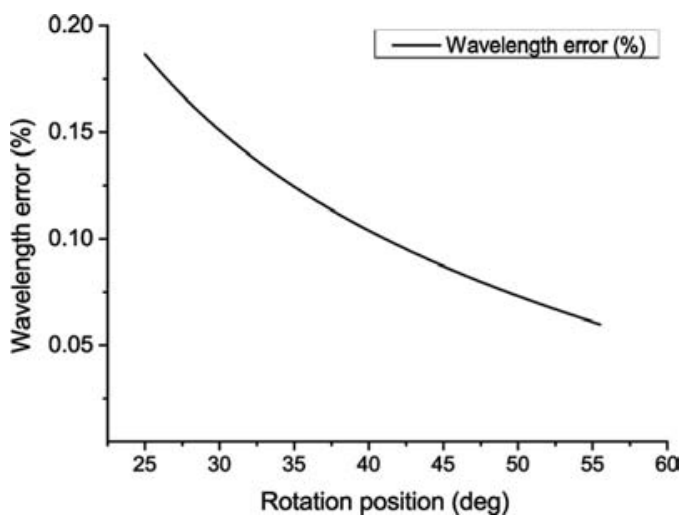

Fig. 9. The relationship between the wavelength error caused by the VHG mechanical rotation part and the particular rotational position.

Table 1. Diffraction efficiency of the volume holographic grating at 488, 532 and $633 \mathrm{~nm}$.

\begin{tabular}{lccc}
\hline Wavelength (nm) & 488 & 532 & 633 \\
Efficiency (\%) & 8.09 & 4.10 & 2.83 \\
\hline
\end{tabular}

to the target wavelength, and $\Delta \theta$ is the absolute accuracy of the VHG mechanical rotation part. The wavelength error of the proposed system, introduced by the absolute accuracy at the particular rotational position, is plotted in Fig. 9. During the experiment, the peak emission wavelengths of each kind of QDs are chosen as the target wavelength channel of switching. The maximum wavelength error is less than $0.8 \mathrm{~nm}$, while the bandwidth of the fluorescence is larger than $40 \mathrm{~nm}$. Therefore, the wavelength error caused by the VHG rotation part is much smaller than the fluorescence bandwidth.

The diffraction efficiencies of the VHG at 488, 532 and $633 \mathrm{~nm}$ are respectively measured and listed in Table 1. In spite of the relatively low diffraction efficiency of the self-recorded VHG in our configuration, fluorescence images with high contrast are obtained at low fluorescence levels. The available incident angle on the hologram is between $25.0^{\circ}$ and $55.5^{\circ}$ due to the mechanical constraints on the incident angle of the camera, which corresponds to a spectral coverage of $392 \mathrm{~nm}$ to $755 \mathrm{~nm}$.

\section{Conclusions}

In conclusion, we have proposed a compact and cost effective VHI method for fluorescence molecular imaging. This spectral selective VHI system can 
operate in the wavelength-scanning mode within a wide wavelength range by continuously switching the rotational position of single transmission volume holographic grating, instead of rotating bulky fluorescence filter wheel or using expensive spectrum selective components. This imaging system can be a flexible tool with which to obtain the accurate three dimensional sub-millimeter information as well as the spectral information of different fluorophores in diffusive medium by performing spectral selective fluorescence imaging. Besides, the spectral selective imaging system can be integrated with existing imaging systems to detect fluorescent targets with unknown wavelength features. Future works include improving the imaging quality of the VHI system by combing different illumination and imaging schemes and enhancing the performance of the reconstructions algorithms.

\section{Acknowledgments}

This work is supported by the National Basic Research Program of China (973) under Grant No. 2011CB707701; the National Natural Science Foundation of China under Grant Nos. 61361160418, 61322101, 81227901, 81271617, and 61401246; the National Major Scientific Instrument and Equipment Development Project under Grant No. 2011YQ030114. The authors thank Drs. Yuan Luo and Hsi-Hsun Chen, in the Center for Optoelectronic Biomedicine of National Taiwan University, for discussions and providing the single transmission volume holographic grating.

\section{References}

1. F. Liu, X. Cao, W. He, J. Song, Z. Dai, B. Zhang, J. Luo, Y. Li, J. Bai, "Monitoring of tumor response to cisplatin by subsurface fluorescence molecular tomography," J. Biomed. Opt. 17, 04050410405043 (2012).

2. T. Lister, P. A. Wright, P. H. Chappell, "Optical properties of human skin," J. Biomed. Opt. 17, 0909011-09090115 (2012).

3. D. A. Boas, D. H. Brooks, E. L. Miller, C. A. DiMarzio, M. Kilmer, R. J. Gaudette, Q. Zhang, "Imaging the body with diffuse optical tomography," IEEE Signal Process. Magaz. 18, 57-75 (2001).

4. N. Deliolanis, T. Lasser, D. Hyde, A. Soubret, J. Ripoll, V. Ntziachristos, M. Lesaffre, F. Jean,
F. Ramaz, A. C. Boccara, "Free-space fluorescence molecular tomography utilizing $360^{\circ}$ geometry projections," Opt. Lett. 32, 382 (2007).

5. M. J. Niedre, R. H. de Kleine, E. Aikawa, D. G. Kirsch, R. Weissleder, V. Ntziachristos, "Early photon tomography allows fluorescence detection of lung carcinomas and disease progression in mice in vivo," Proc. Nat. Acad. Sci. 105, 19126-19131 (2008).

6. A. J. Chaudhari, F. Darvas, J. R. Bading, R. A. Moats, P. S. Conti, D. J. Smith, S. R. Cherry and R. M. Leahy, "Hyperspectral and multispectral bioluminescence optical tomography for small animal imaging," Phys. Med. Biol. 50, 5421-5441 (2005).

7. A. Corlu, "Multi-spectral and fluorescence diffuse optical tomography of breast cancer," Ph.D. thesis, University of Pennsylvania (2007).

8. J. Vila-Francés, J. Calpe-Maravilla, L. GómezChova, J. Amorós-López, "Analysis of acousto-optic tunable filter performance for imaging applications," Opt. Eng. 49, 113203-113203-9 (2010).

9. Z. Arsov, I. Urbančič, M. Garvas, D. Biglino, A. Ljubetič, T. Koklič, J. Štrancar, "Fluorescence microspectroscopy as a tool to study mechanism of nanoparticles delivery into living cancer cells," Biomed. Opt. Express 2, 2083-2095 (2011).

10. P. Favreau, C. Hernandez, A. S. Lindsey, D. F. Alvarez, T. Rich, P. Prabhat, S. J. Leavesley, "Thin-film tunable filters for hyperspectral fluorescence microscopy," J. Biomed. Opt. 19, 011017011017 (2014).

11. A. Sinha, G. Barbastathis, "Volume holographic imaging for surface metrology at long working distances," Opt. Express 11, 3202-3209 (2003).

12. M. Balberg, D. J. Brady, "Confocal microscopy with a volume holographic filter," Opt. Lett. 24, 811-813 (1999).

13. D. Psaltis, G. Barbastathis, "Real-time spectral imaging in three spatial dimensions," Opt. Lett. 27, 854-856 (2002).

14. A. Sinha, G. Barbastathis, W. Liu, D. Psaltis, "Imaging using volume holograms," Opt. Eng. 43, 1959-1972 (2004).

15. N. Gat, "Imaging spectroscopy using tunable filters: a review," 4056, 50-64 (2000). 10.1117/12.381686 doi: $10.1117 / 12.381686$.

16. A. L. Glebov, O. Mokhun, A. Rapaport, S. Vergnole, V. Smirnov, L. B. Glebov, "Volume bragg gratings as ultra-narrow and multiband optical filters," in Proc. SPIE Photonics Europe Int. Soc. Opt. Photon., pp. 84280C-84280C-11 (2012).

17. G. Barbastathis, The transfer function of volume holographic optical systems, Photorefractive Materials and their Applications, Vol. 3, pp. 51-76, Springer, Newyork (2007). 
18. A. Sinha, G. Barbastathis, "N-ocular volume holographic imaging," Appl. Opt. 43, 5784-5795 (2004).

19. Y. Luo, I. K. Zervantonakis, S. B. Oh, R. D. Kamm, G. Barbastathis, "Spectrally resolved multidepth fluorescence imaging," J. Biomed. Opt. 16, 096015096015-5 (2011).

20. G. V. Orsinger, J. M. Watson, M. Gordon, A. C. Nymeyer, E. E. de Leon, J. W. Brownlee, K. D. Hatch, S. K. Chambers, J. K. Barton, R. K. Kostuk, "Simultaneous multiplane imaging of human ovarian cancer by volume holographic imaging," $J$. Biomed. Opt. 19, 036020-036020 (2014).

21. Y. Luo, V. R. Singh, D. Bhattacharya, E. Yew, J. C. Tsai, S. L. Yu, H. H. Chen, J. M. Wong, P. Matsudaira, Peter T. C. So, "Talbot holographic illumination nonscanning (thin) fluorescence microscopy." Laser 83 Photonics Rev. 8(5), L71L75, (2014).

22. Hsi-H. Chen, V. R. Singh, Y. Luo, "Speckle-based volume holographic microscopy for optically sectioned multi-plane fluorescent imaging," Opt. Express 23(6), 7075-7084, (2015).

23. Z. Li, D. Psaltis, W. Liu, W. R. Johnson, G. Bearman, "Volume holographic spectral imaging," in "Proc. SPIE," Vol. 5694 Proc. SPIE, pp. 33-40 (2005).

24. F. Liu, X. Liu, D. Wang, B. Zhang, Jing Bai, "A parallel excitation based fluorescence molecular tomography system for whole-body simultaneous imaging of small animals," Ann. Biomed. Eng. 38(11), 3440-3448, (2010).

25. J. Ripoll, V. Ntziachristos, R. Carminati, M. NietoVesperinas, "Kirchhoff approximation for diffusive waves," Phys. Rev. E 64, 051917 (2001).

26. M. Hanke, C. W. Groetsch, "Nonstationary iterated tikhonov regularization," J. Optimiz. Theory Appl. 98, 37-53 (1998).

27. D. Wang, H. Qiao, X. Song, Y. Fan, D. Li, "Fluorescence molecular tomography using a twostep three-dimensional shape-based reconstruction with graphics processing unit acceleration," Appl. Opt. 51, 8731-8744 (2012).

28. Z. Shen, J. Li, L. Huang, F. Luo, Y. Luo, D. Zhang, Y. Long. "Research on imaging spectrometer using lc-based tunable filter", 8515, 85150H-85150H-6, (2012). 10.1117/12.928563, doi: 10.1117/12.928563.

29. J. Shi, F. Liu, J. Zhang, J. Luo, Jing Bai, "Fluorescence molecular tomography reconstruction via discrete cosine transform-based regularization ", J. Biomed. Opt. 20 (5), 055004-055004, (2015).

30. J. W. Goodman, Introduction to Fourier optics, Roberts and Company Publishers US (2005).

31. J. Shi, B. Zhang, F. Liu, J. Luo, J. Bai, "Efficient L1 regularization-based reconstruction for fluorescent molecular tomography using restarted nonlinear conjugate gradient". Opt. Lett. 38 (18), 3696-3699, (2013). 